\title{
RUNX2 controls human IPO8 basal transcription in Saos-2 cells
}

\author{
JIANJUN XIONG ${ }^{1}$, ZHIHONG HU $^{1}$, TING WANG ${ }^{1}$, XIAOYUAN XU $^{1}$, \\ JIANYUN LIU ${ }^{2}$, PING WU ${ }^{2}$, XIANGXIN CHE ${ }^{1}$ and WEIDONG LI ${ }^{2}$ \\ ${ }^{1}$ Department of Pharmacology, College of Basic Medical Science; ${ }^{2}$ Key Laboratory of Jiangxi for the Systems Bio-medicine, \\ Jiujiang University, Jiujiang, Jiangxi 332000, P.R. China
}

Received June 18, 2015; Accepted May 9, 2016

DOI: $10.3892 / \mathrm{mmr} .2016 .5356$

\begin{abstract}
Runt-related transcription factor 2 (RUNX2) is a vital regulatory factor that controls osteoblast-specific gene expression; however, RUNX2-regulated genes in human mesenchymal stem cells (hMSCs) remain to be fully elucidated. In the present study, chromatin immunoprecipitation (ChIP)-on-chip analysis of RUNX2 in hMSCs demonstrated that importin 8 (IPO8) may be a novel target gene. The $5^{\prime}$ flanking region of the IPO8 gene, which is $\sim 3,300 \mathrm{bp}$ in length, was cloned and inserted into the pGL3-basic luciferase reporter vector. The results of dual luciferase reporter assays indicated that this segment possessed strong basal promoter activity. Furthermore, the RUNX2 binding site, which encompasses positions -496 to $-501 \mathrm{bp}$, was required to achieve maximal IPO8 promoter activity in Saos-2 human osteosarcoma cells. In addition, ChIP analysis indicated that RUNX2 uniquely binds to this specific IPO8 sequence motif. Cells with a knockdown in RUNX2 expression exhibited downregulated IPO8 transcription. Finally, synchronization of IPO8 and RUNX2 expression was observed in Saos-2 cells cultured in osteoblast-induction medium. Taken together, these results indicated that RUNX2 regulates IPO8 gene transcription, and may have a contributory role in osteoblast differentiation.
\end{abstract}

\section{Introduction}

Human mesenchymal stem cells (hMSCs) are characteristically multipotent, and are therefore considered potential cellular therapies for tissue regeneration $(1,2)$. In response to osteoblast-induction medium, hMSCs can differentiate into an osteogenic lineage and exhibit a typical osteoblast phenotype. Runt-related transcription factor 2 (RUNX2), which is an important transcription factor that regulates osteogenic gene expression, is activated during the differentiation of hMSCs into osteoblasts and triggers the expression of various

Correspondence to: Professor Weidong Li, Key Laboratory of Jiangxi for the Systems Bio-medicine, Jiujiang University, 17 Lufeng Road, Jiujiang, Jiangxi 332000, P.R. China

E-mail: 1wd626518@163.com

Key words: RUNX2, IPO8, transcription, Saos-2 cells osteoblast-associated markers, including collagen I, alkaline phosphatase and osteocalcin $(3,4)$; therefore, has a key role in bone formation regulation. Previous studies have reported that RUNX2 binds to the promoter of several genes, which are predominantly expressed in osteoblasts $(5,6)$; however, RUNX2-regulated genes in hMSCs have yet to be fully elucidated.

The passage of molecules into the nucleus is facilitated by the activity of nuclear pore complexes (NPCs) (7). The classical nuclear import pathway involves two components, namely importin- $\beta$, and the adaptor protein, importin- $\alpha$. The binding of importin- $\beta$ to importin- $\alpha$ triggers a conformational change in importin- $\alpha$, which increases its affinity to cargo proteins that possess a nuclear localization signal (NLS) (8). Importin- $\beta$ is the largest evolutionarily conserved family of nuclear transport receptors. In addition to its role in the transport of nucleocytoplasmic materials, importin- $\beta$ is considered a global regulatory factor of transport-associated cellular functions during the cell cycle (9).

The importin 8 (IPO8) gene, which belongs to the importin- $\beta$ family and is located at chromosomal region 12p11.21, encodes a 1,037-amino acid protein (10). Previous studies have reported that the IPO8 gene is one of the most commonly used reference genes for clinicopathological analysis (11-13). However, other studies have revealed that IPO8 interacts in an RNA-dependent manner with Argonaute (AGO) proteins, which are involved in microRNA (miRNA) processing and function (14). Furthermore, HeLa and HEK293 cells with an IPO8 knockdown exhibited a redistribution of $\mathrm{AGO}$, which was originally located in the nucleus, into the cytoplasm, which in turn led to a moderate increase in target mRNAs (14). These findings indicate that IPO8 may possess specific functions that are yet to be elucidated; however, information on the control of IPO8 gene expression is currently inadequate.

The present study conducted a RUNX2 chromatin immunoprecipitation (ChIP)-on-chip analysis in hMSCs, which revealed IPO8 as a target gene of RUNX2. Promoter deletion and mutation assays demonstrated that RUNX2 is essential in attaining the maximum level of IPO8 promoter activity in Saos-2 human osteosarcoma cells. In addition, a ChIP assay confirmed the specific binding of RUNX2 to the IPO 8 promoter. The present study also confirmed the synchronization of IPO8 and RUNX2 expression during the process of osteoblast differentiation. The present study is the first, to the best of our knowledge, to provide insight into understanding 
the transcriptional regulation of $I P O 8$ by RUNX2, in which IPO8 may be involved in osteoblast differentiation.

\section{Materials and methods}

ChIP. hMSCs (American Type Culture Collection, Manassas, VA, USA) were chemically cross-linked with $1 \%$ formaldehyde. The fixed cells were washed twice with phosphate-buffered saline and were lysed using a lysis buffer [0.1\% sodium dodecyl sulfate (SDS), $0.5 \%$ Triton X-100, $20 \mathrm{mM}$ Tris-HCl, $\mathrm{pH}$ 8.1] that contained $150 \mathrm{mM} \mathrm{NaCl}$ and a protease inhibitor. The lysed cells were subsequently subjected to sonication in ice water. The resulting sonicated fragments were within the size range of 200-1,000 bp. Following sonication, the samples were centrifuged at $13,000 \mathrm{xg}$ for $10 \mathrm{~min}$ at $4^{\circ} \mathrm{C}$, and the supernatant was pre-absorbed by $50 \mu \mathrm{l}$ protein $\mathrm{G}$ beads and was incubated with magnetic beads conjugated to RUNX2 antibodies [RUNX2 (C-19) cat. no. sc-8566; Santa Cruz Biotechnology, Inc., Dallas TX, USA; or cat. no. OALA04014; Aviva Systems Biology Corporation, San Diego, CA, USA] overnight at $4^{\circ} \mathrm{C}$. IgG (H-270) antibody (cat. no. sc-66931, Santa Cruz Biotechnology, Inc.) served as a negative control. The magnetic beads were then rinsed four times with lysis buffer, twice with $\mathrm{LiCl}$ buffer, and three times with Tris-EDTA buffer. The bound immunocomplex was eluted by adding $300 \mu \mathrm{l}$ of fresh elution buffer $[10 \mathrm{mM}$ Tris; $1 \mathrm{mM}$ EDTA, (pH 8.0)]. Subsequently, $20 \mu 15 \mathrm{M} \mathrm{NaCl}$ was mixed with the eluted product, which was incubated at $65^{\circ} \mathrm{C}$ overnight to reverse the crosslinking. Immunoprecipitated genomic DNA was then purified and dissolved in EB buffer for ChIP-on-chip analysis.

The immunoaffinity-enriched DNA was subjected to amplification using a whole genome amplification kit (WGA kit; Sigma-Aldrich, St. Louis, MO, USA). The amplified products were submitted to purification using a QIAquick Polymerase Chain Reaction (PCR) Purification kit (Qiagen GmbH, Hilden, Germany). To assess ChIP enrichment, qPCR for known specific transcription factor binding sites was performed

ChIP-on-chip assays. To label DNA, the NimbleGen Dual-Color DNA Labeling kit (Roche NimbleGen, Inc., Madison, WI, USA) was used, according to the manufacturer's protocol. DNA $(\sim 1 \mu \mathrm{g})$ from each sample was then incubated for $10 \mathrm{~min}$ at $98^{\circ} \mathrm{C}$, with $\sim 1$ OD Cy5-9mer primer (IP sample) or Cy3-9mer primer (input sample; Roche Applied Science, Penzberg, Germany). Subsequently, 100 pmol deoxynucleoside triphosphates were mixed with $100 \mathrm{U}$ Klenow fragment (New England Biolabs, Ipswich, MA, USA), which was incubated at $37^{\circ} \mathrm{C}$ for $2 \mathrm{~h}$. The labeling reaction was terminated following the addition of a 0.1 volume of $0.5 \mathrm{M}$ EDTA, which was followed by DNA purification and precipitation using isopropanol/ethanol [precipitation with isopropanol $(12,000 \times \mathrm{g}$ for $15 \mathrm{~min})$; washing with $1 \mathrm{ml} 70 \%$ ethanol $(12,000 \mathrm{x} \mathrm{g}$ for $5 \mathrm{~min})]$. The microarrays were then hybridized to $4 \mu \mathrm{g}$ Cy3/5-labeled DNA dissolved in NimbleGen hybridization buffer/hybridization component $\mathrm{A}$ at $42^{\circ} \mathrm{C}$ for 16-20 $\mathrm{h}$ in a hybridization chamber (Roche NimbleGen, Inc.). Washing was performed using the NimbleGen Wash Buffer kit (Roche NimbleGen, Inc.). To conduct array hybridization, the Human ChIP-chip 3X720K RefSeq Promoter Array (Roche NimbleGen, Inc.) was used.
Cell cultures. The Saos-2 human osteosarcoma cell line (American Type Culture Collection) was cultured in McCoy's 5A (Gibco; Thermo Fisher Scientific, Inc. Waltham, MA, USA) supplemented with 15\% (v/v) fetal bovine serum (Invitrogen; Thermo Fisher Scientific, Inc.) at $37^{\circ} \mathrm{C}$ in an atmosphere containing $5 \% \mathrm{CO}_{2}$. Cells were passaged according to standard cell culture techniques.

5' rapid amplification of cDNA ends (RACE). A 5' RACE system was used, according to the manufacturer's protocol (Takara Biotechnology Co., Ltd., Dalian, China). Briefly, $10 \mu \mathrm{g}$ total RNA was extracted from Saos-2 cells using TRIzol Reagent (Invitrogen; Thermo Fisher Scientific, Inc.) and was treated with calf intestinal phosphatase, in order to remove any free 5'-phosphate groups. Tobacco acid pyrophosphatase was used to specifically detach the cap structure from the full-length mRNA, which in turn isolates the 5'-monophosphate segment. An RNA oligonucleotide adaptor was then attached to the newly de-capped mRNA strand at the 5' end using a T4 RNA ligase. Using the ligated RNA as a template, IPO 8 cDNA synthesis via reverse transcription was conducted using Murine Moloney Virus (M-MLV) reverse transcriptase and random primers (reaction volume, $20 \mu \mathrm{l}$ per well). Briefly, $400 \mathrm{ng}$ total RNA was reverse transcribed using the PrimeScript ${ }^{\mathrm{TM}} \mathrm{RT}$ reagent kit with gDNA Eraser (Takara Biotechnology Co., Ltd.). The protocol was as follows: $25^{\circ} \mathrm{C}$ for $5 \mathrm{~min}, 42^{\circ} \mathrm{C}$ for $1 \mathrm{~h}$ and $70^{\circ} \mathrm{C}$ for $5 \mathrm{~min}$. The generated cDNA was subsequently amplified via nested PCR using the LA Taq DNA polymerase (Takara Biotechnology Co., Ltd.). The reaction conditions were as follows: $94^{\circ} \mathrm{C}$ Initial denaturation $(5 \mathrm{~min}) ; 94^{\circ} \mathrm{C}$ denaturation for $30 \mathrm{sec}$ and $56^{\circ} \mathrm{C}$ annealing for $30 \mathrm{sec}$, and $72^{\circ} \mathrm{C}$ elongation for $40 \mathrm{sec}$, for 30 cycles. The gene-specific antisense outer primer 5'-CTTCTTACACTTCCACCATA-3' (+789 to +770) and inner primer 5'-CACCAGTTGATACAGGCATA-3' (+471 to +452) were based on the IPO 8 cDNA sequence. The PCR products were then analyzed by agarose gel electrophoresis and were cloned into the PMD-18T vector (Takara Biotechnology Co., Ltd.) prior to direct sequencing (Invitrogen Biotechnology Co. Ltd., Shanghai, China), in order to identify the transcription start site (TSS).

Plasmid constructs. PCR analyses were conducted using Pyrobest DNA polymerase (Takara Biotechnology Co., Ltd.). Briefly, chromosome DNA was isolated according to the manufacturer's instructions (Tiangen Biotech, Co., Ltd., Beijing, China). A series of deletion DNA fragments consisting of the $5^{\prime}$ flanking region of the IPO8 gene were PCR amplified (reaction volume, $20 \mu 1$ per well; initial denaturation, $94^{\circ} \mathrm{C}$ for $5 \mathrm{~min}$; denaturation, $94^{\circ} \mathrm{C}$ for $30 \mathrm{sec}$; annealing, $56^{\circ} \mathrm{C}$ for $30 \mathrm{sec}$; elongation, $72^{\circ} \mathrm{C}$ for $40 \mathrm{sec}$, for 30 cycles) using genomic DNA extracted from Saos- 2 cells. The following primers were used: Upstream, 5'-TTCCAC GCGTAATCAAAGGGTTAGGAATGT-3' for P-3302/+134, 5'-TTCCACGCGTGTGGGTCAAGG CTAGAGTTA-3' for P-2193/+134, 5'-TTCCACGCGTTG TGGCTCGCTTCTTCAGTG-3' for P-1337/+134, 5'-TTCCAC GCGTGTGTCAGTCCTCACCTAGGT-3' for P-732/+134, 5'-TTCCACGCGTCGATGCCCATACAG TTCTCG-3' for P-330/+134, 5'-TTCCACGCGTGACGG GAGGCGGTCATAGC-3' for P-97/+134, and the 
downstream primer was 5'-AAGGAGATCTCGACC CCTGGATTACCTCAC-3' for all. The PCR products were subjected to gel purification and were then subcloned into the pGL3-basic firefly luciferase vector (Promega Corporation, Madison, WI, USA). Site-directed mutagenesis, which inactivates the RUNX2 binding site, was conducted, according to the protocol of the MutanBEST kit (Takara Biotechnology Co., Ltd.). All constructs were confirmed by DNA sequencing.

Transient transfections and dual luciferase reporter assays. Cells at a density of $5 \times 10^{4}$ cells per well were seeded onto 24-well culture dishes and transiently transfected using Fugene 6.0 (Promega Corporation), according to the manufacturer's protocol. Each well received $1.0 \mu \mathrm{g} I P O 8$ reporter plasmids and $0.2 \mu \mathrm{g}$ Renilla luciferase construct (pRL-TK) as an internal control. All transfection experiments were conducted in triplicate. A total of $24 \mathrm{~h}$ post-transfection, the cells were lysed and used for the dual luciferase assays (DualLuciferase ${ }^{\circledR}$ Reporter assay system; Promega Corporation).

RNA interference. Cells were transfected with RUNX2 short hairpin (sh)RNA lentiviral particles (sc-37145-V; Santa Cruz Biotechnology, Inc.) or control shRNA lentiviral particles (sc-108080; Santa Cruz Biotechnology, Inc.), according to the manufacturer's protocol. Briefly, $5 \times 10^{5}$ target cells were plated in a 6-well plate alongside $2 \mathrm{ml}$ complete optimal medium with siRNA Transfection Reagent (Invitrogen; Thermo Fisher Scientific, Inc.), and were incubated overnight at $37^{\circ} \mathrm{C}$. The medium was then removed from the wells and was replaced with $2 \mathrm{ml} 5 \mu \mathrm{g} / \mathrm{ml}$ polybrene media mixture and $2 \mu 1 \mathrm{shRNA}$ lentiviral particles per well. A total of $48 \mathrm{~h}$ post-infection, the shRNA-infected cells were harvested and subjected to quantitative (q)PCR or western blot analysis.

qPCR. Saos-2 cells treated with osteogenic differentiation media (Invitrogen; Thermo Fisher Scientific, Inc.) were harvested and total RNA was extracted, as described previously. RNA ( $2 \mu \mathrm{g}$ ) was used to generate cDNA using M-MLV reverse transcriptase (Promega Corporation). qPCR analysis was conducted using SYBR Green PCR Master Mix (Qiagen $\mathrm{GmbH}$ ) and an ABI 7500 Real-Time PCR system (Applied Biosystems; Thermo Fisher Scientific, Inc.). The PCR reaction conditions were as follows: $94^{\circ} \mathrm{C}$ Initial denaturation (10 min); $94^{\circ} \mathrm{C}$ denaturation for $15 \mathrm{sec}$ and $60^{\circ} \mathrm{C}$ annealing and elongation for $60 \mathrm{sec}$, for 40 cycles. The quantitative data were determined using the $2^{-\Delta \Delta \mathrm{Cq}}$ method (15). The primer pairs (Sangon Biotech Co., Ltd., Shanghai, China) used in the present study were as follows: IPO8, forward 5'-TGTTCAGCT CCTTCCTGATTC-3', reverse 5'-CTTCTTACACTTCCA CCATAC-3'; RUNX2, forward 5'-GCCTTCAAGGTGGTA GCCC-3', reverse 5'-AAGGTGAAAC TCTTGCCTCGTC-3'. glyceraldehyde 3-phosphate dehydrogenase $(G A P D H)$, forward 5'-AGAAGGCTGGGGCTCATTTG-3' and reverse 5'-AGGGGCCATCCACAGTCTTC-3'.

Western blot analysis. Cell lysis was conducted using $1 \mathrm{X}$ SDS sample buffer (1\% SDS). Proteins (25 $\mu 1)$ were separated by $10 \%$ SDS-polyacrylamide gel electrophoresis, and were electroblotted onto a nitrocellulose membrane. The membranes were then blocked with $5 \%$ non-fat milk in

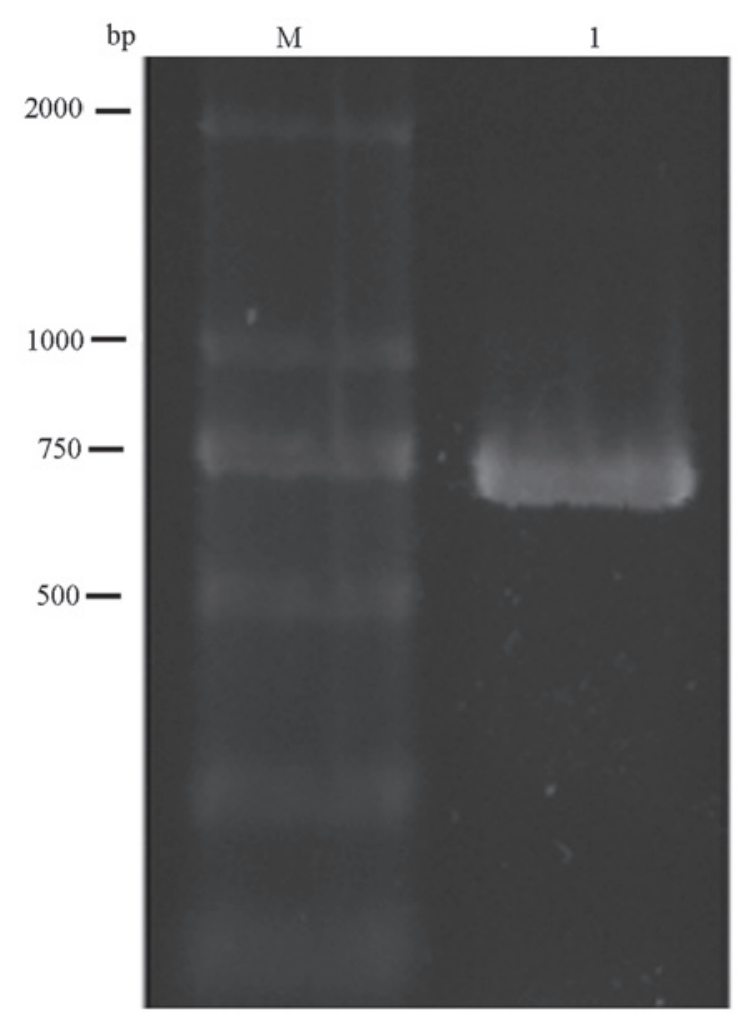

Figure 1. Determination of the transcription start site of importin 8 (IPO8). Total RNA isolated from Saos-2 cells was subjected to a 5' rapid amplification of cDNA ends (RACE) assay using a $5^{\prime}$ random primer mix and 3' IPO8-specific primers. Following amplification, the 5' RACE products were visualized by $1.5 \%$ agarose gel electrophoresis and were stained with ethidium bromide. Lane M represents a DL2000 DNA ladder (Takara Biotechnology Co., Ltd.) and lane 1 represents the 5' RACE IPO8 products.

tris-buffered saline for $2 \mathrm{~h}$, and hybridized to an anti-RUNX2 mouse antibody (cat. no. sc-390715 and dilution, 1:500; Santa Cruz Biotechnology, Inc.), anti-IPO8 rabbit antibody (cat. no. ab72109 and dilution, 1:1,000; Abcam, Cambridge, UK), or anti-GAPDH mouse antibody (cat. no. sc-365062 and dilution, 1:1,000; Santa Cruz Biotechnology, Inc.) overnight at $4^{\circ} \mathrm{C}$. Following washing, the membranes were incubated with horseradish peroxidase-conjugated rabbit anti-mouse IgG (dilution, 1:10,000; cat. no. sc-358920; Santa Cruz Biotechnology, Inc.) or goat anti-rabbit IgG (dilution, 1:10,000; cat. no. sc-2004; Santa Cruz Biotechnology, Inc.) secondary antibodies for $1 \mathrm{~h}$ at room temperature. The blots were developed using the Pierce enhanced chemiluminescence system (Pierce Biotechnology, Inc., Rockford, IL, USA), according to the manufacturer's protocol.

Statistical analysis. Data are presented as the mean \pm standard error of the mean. Comparisons among three or more experimental groups were performed by one-way analysis of variance using SPSS 11.0 software (SPSS, Inc., Chicago, IL, USA) and $\mathrm{P}<0.05$ was considered to indicate a statistically significant difference.

\section{Results}

RUNX2 targets the IPO8 gene in hMSCs. RUNX2 is considered a key transcription factor that binds to the promoter 
region of various genes, which are predominantly expressed in osteoblasts. In the present study, two independent biological replicates of the ChIP-on-chip experiment were performed on hMSCs using two types of anti-RUNX2 antibodies. To assess ChIP enrichment, qPCR for known specific transcription factor binding sites was performed on input DNA, ChIP DNA and mock IP DNA from each sample. The promoter regions of osterix were amplified from RUNX2-immunoprecipitated DNA, confirming chromatin enrichment by ChIP. For array hybridization, the Roche NimbleGen Human ChIP-chip 3X720K RefSeq Promoter Array was used, which is a single array design that includes 22,542 gene promoter regions (from $-3,200$ to +800 bp of the TSSs) covering $\sim 720,00050-75 \mathrm{mer}$ probes that are spaced apart by $\sim 100 \mathrm{bp}$, dependent on the nucleotide sequence of the segment. Notably, RUNX2 binding was observed for the human IPO8 gene in both types of RUNX2 ChIP assay.

Analysis of promoter activity within the 5' flanking region. To characterize the IPO 8 gene TSS, a 5' RACE system was used to amplify the 5' terminus of IPO 8 cDNA generated from the mRNA of Saos-2 cells. Sequencing of the 700-bp PCR product generated by $5^{\prime}$ RACE identified a single TSS $(+1)$, which was located -333 bp upstream of the initiation codon (Fig. 1).

Based on the results of the TSS analysis, a 3,436-bp region $(-3,302$ to $+134 \mathrm{bp})$ that encompasses the TSS was amplified from human genomic DNA and inserted into the promoter-deleted pGL3-basic firefly luciferase vector. Post-transfection into Saos-2 cells, the P-3302 construct exhibited significant promoter activity that was $>100$-fold greater than the activity of the control plasmid pGL3-basic, thus suggesting that the $-3,302$ to +134 bp region had strong basal promoter activity. To identify the regions controlling IPO 8 promoter activity, a set of 5 ' truncated constructs were inserted in the upstream region of the luciferase reporter gene of the pGL3-basic vector. No significant luciferase activity differences were detected between the P-2193 and P-3302 constructs, suggesting the absence of activation or repression motifs between -2,193 and -3,302 bp. In addition, the constructs P-1337 and P-732 exerted similar promoter activities, which were the highest among all of the constructs in Saos-2 cells. Further truncation from bp -732 to -330 resulted in significant decreases in luciferase activity. These results indicated that the region -732 to -330 bp possessed the critical element(s) necessary for maximum activity of the IPO8 promoter (Fig. 2A).

TRANSFAC analysis (http://www.gene-regulation.com) demonstrated that the region between -732 and -330 bp contained a TGTGGT sequence (-496 to $-501 \mathrm{bp}$ ), which has been identified as a component of the binding site for RUNX2 $(16,17)$. To evaluate the role of this DNA motif in IPO8 gene transcription, site-directed mutation and deletion was introduced into the RUNX2 binding site and promoter activity was determined. As presented in Fig. 2B, disruption of the RUNX2 binding site reduced luciferase gene expression by $50 \%$ compared with the wild-type.

RUNX2 binds to the promoter region of the IPO8 gene in Saos-2 cells. To determine whether cellular RUNX2 binds to the IPO 8 promoter region in Saos-2 cells, ChIP was performed using RUNX2 (C-19) antibody, which specifically reacts
A Relative luciferase activity
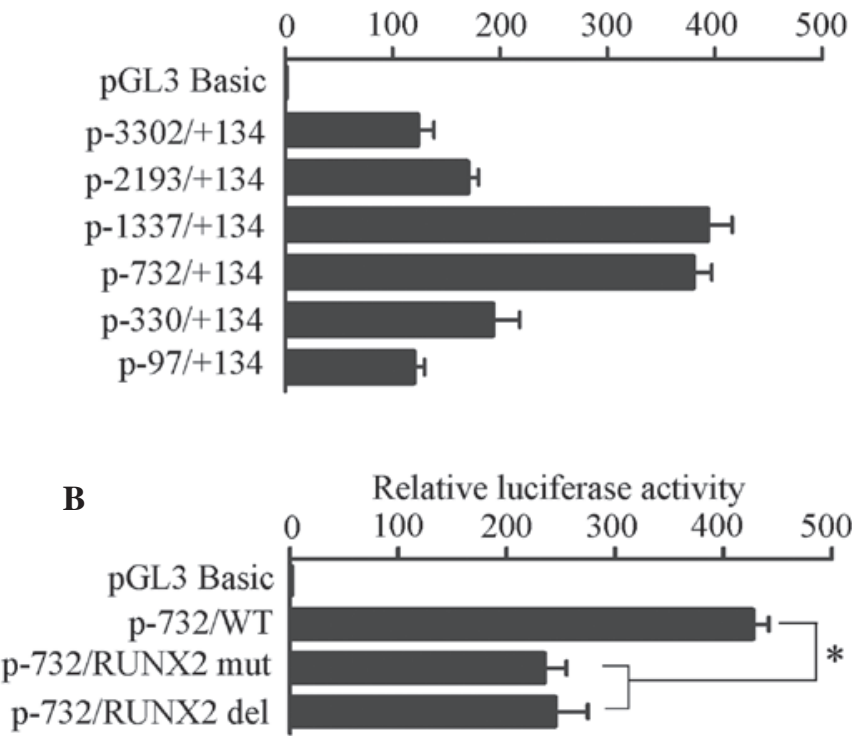

Figure 2. Functional analysis of importin 8 (IPO8) promoter constructs (A) Functional analysis of IPO8 promoter activity using 5' deletion constructs in Saos-2 cells. Bars show fold-increase in luciferase activity for the IPO8 promoter constructs cloned into the pGL3-basic vector compared with that of the promoter-deleted pGL3-basic vector. The numbers on the left indicate the 5'-ends of the constructs relative to the transcription start site. Data are presented as the mean \pm standard error of the mean of triplicates from at least three independent experiments. (B) Luciferase activity expressed by the runt-related transcription factor 2 (RUNX2) site-directed mutant (mut) or deletion (del) mutant relative to pGL3-basic activity. Each bar represents the mean \pm standard deviation of three independent experiments. ${ }^{*} \mathrm{P}<0.05$ vs. control. WT, wild-type.

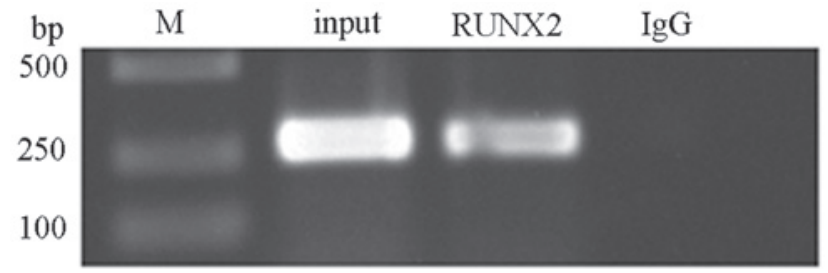

Figure 3. Identification of runt-related transcription factor 2 (RUNX2) binding to the importin 8 (IPO8) promoter. To determine whether cellular RUNX2 bound to the IPO 8 promoter region, chromatin immunoprecipitation (ChIP) was performed using an antibody targeting human RUNX2. Immunoprecipitation of cross-linked chromatin from Saos-2 cells with anti-RUNX2 antibody was followed by polymerase chain reaction amplification of the region between -662 and -424 bp. The ChIP assays were repeated three times using independent preparations. M, DNA ladder; IgG, immunoglobulin G.

to human RUNX2. Immunoprecipitation of cross-linked chromatin with the anti-RUNX2 antibody, followed by PCR amplification of the region (the primers were as follows: 5'-TTC ACTTCGCCCATCCCT-3'; 5'-CATTCATTCATTCGCTTT CA-3'), determined that the endogenous RUNX2 polypeptide was indeed bound to this particular segment (size, $239 \mathrm{bp}$ ) of the IPO 8 promoter of Saos-2 cells (Fig. 3).

RUNX2 has a role in regulating IPO 8 transcription. To examine the involvement of RUNX2 in the transcriptional regulation of IPO8, RUNX2 expression was suppressed using lentivirus-mediated shRNA. Saos-2 cells were infected with 


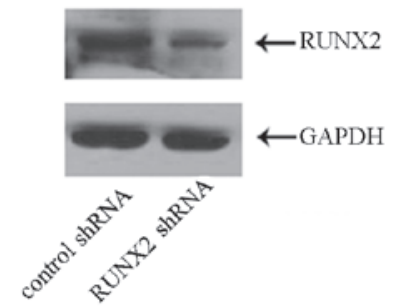

B

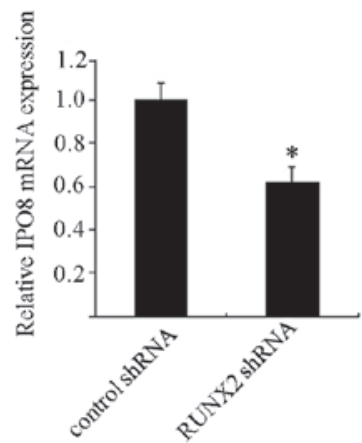

C

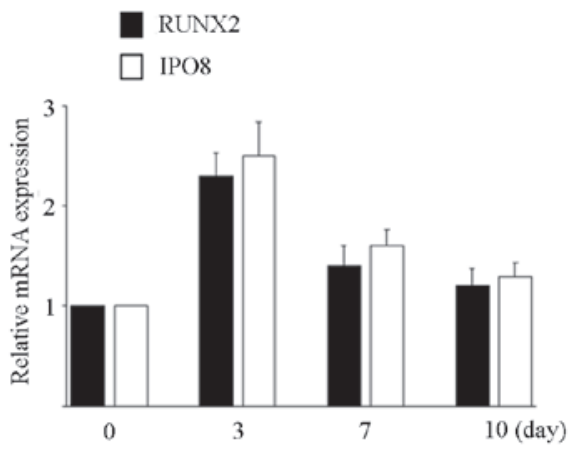

Figure 4. Effects of runt-related transcription factor 2 (RUNX2) knockdown on importin 8 (IPO8) transcription. (A) Saos-2 cells were transfected with RUNX2 short hairpin (sh)RNA, or scrambled shRNA as a control. After $24 \mathrm{~h}$, total protein was collected for assessment of protein levels by western blotting. The expression levels of RUNX2 were normalized to those of glyceraldehyde 3-phosphate dehydrogenase (GAPDH). (B) Saos-2 cells were transfected with RUNX2 shRNA or control shRNA. After 24 h, IPO 8 mRNA expression was detected by quantitative polymerase chain reaction. (C) IPO 8 and $R U N X 2$ mRNA expression levels in Saos-2 cells on days $0,3,7$ and 10 of osteoblast differentiation. Each data point represents the mean \pm standard deviation of three independent samples. ${ }^{*} \mathrm{P}<0.05$ vs. control.

shRNA that specifically targets RUNX2 or control shRNA for $48 \mathrm{~h}$, and were harvested for RUNX2 detection. As shown in Fig. 4A, the protein expression levels of RUNX2 were significantly reduced to $<25 \%$ compared with the control cells treated with a control shRNA. Subsequently, the endogenous IPO 8 mRNA expression levels were evaluated by qPCR. As shown in Fig. 4B, treatment with RUNX2-specific shRNA induced a significant reduction in the IPO 8 mRNA expression by $\sim 50 \%$.

Since IPO8 is the downstream target of RUNX2, it was hypothesized that IPO8 may be involved in osteoblast differentiation. Therefore, RUNX2 and IPO 8 mRNA expression levels were detected in Saos-2 cells following treatment with osteogenic differentiation media. As shown in Fig. 4C, the mRNA expression levels of IPO 8 were significantly increased after 3 days of treatment and subsequently decreased at day 7 . RUNX2 expression was also observed to be in synchronization with this trend.

\section{Discussion}

The present study generated the following key findings: i) IPO8 is a novel target gene of RUNX2 in hMSCs; ii) the IPO8 TSS was identified in Saos-2 cells and characterization of its 5' flanking regions was conducted; iii) the RUNX2 binding site was required for maximum IPO 8 promoter activity; iv) RUNX2 regulated IPO8 transcription; and v) the mRNA expression levels of IPO8 are synchronized with RUNX2 expression during osteoblast differentiation in human osteosarcoma cells.

hMSCs are self-renewing multipotent cells that may potentially be used as cellular therapies for tissue regeneration. Under appropriate conditions, hMSCs have the ability to differentiate into osteoblasts; however, the control of these cellular pathways has yet to be fully established. RUNX2 is a runt-related transcription factor, which is activated during osteoblast differentiation, and triggers the expression of osteoblast-specific markers, including collagen I, alkaline phosphatase and osteocalcin, during the initial stages of osteoblast maturation. Therefore, RUNX2 is considered to have an essential role in the control of bone development $(3,4)$. RUNX2-deficient mice are incapable of developing an ossified skeleton due to an insufficiency in the number of osteoblasts, thus indicating the essential function of RUNX2 in determining the osteoblastic cell fate of MSCs (18). The aim of the present study was to examine the novel target genes regulated by RUNX2 in hMSCs.

ChIP-on-chip is a technique that facilitates the large-scale determination of genomic regions interacting with DNA-binding proteins under physiologically essential in vivo conditions. As a member of the RUNX family, RUNX2 forms heterodimers with core-binding factor subunit $\beta$ and recognizes the consensus sequences PyGPyGGTPy $(16,17)$, thus regulating gene transcription, including that of osterix (19). Previously, genome-wide occupancy analysis of RUNX2 at gene loci in Saos-2 human osteosarcoma cells was performed, which revealed several novel potential target genes, including Talin-1 and cyclic AMP-responsive element-binding protein 3 (20). The present study performed a genome-wide ChIP-on-chip analysis of promoters bound by RUNX2 in hMSCs, the results of which identified a 22,542 gene promoter region (from $-3,200$ to +800 bp of the TSSs), which was completely encompassed by $\sim 720,000$ 50-75 mer probes at intervals of $\sim 100 \mathrm{bp}$, dependent on the sequence of that particular region. In addition, various novel potential target genes regulated by RUNX2 were identified. Among these genes, IPO 8 exhibited a higher maximum score in two types of RUNX2 immunoprecipitation assay, these findings have not been described in previous studies.

IPO 8 belongs to the importin $\beta$ family. The importin- $\alpha / \beta$ complex, in combination with the GTPase ras-related nuclear protein (Ran), mediates nuclear import of proteins that possess a classic NLS. IPO 8 undergoes binding to the NPC. Furthermore, along with RanGTP and Ran binding protein 1, IPO8 prevents GTPase-activating protein induction of Ran GTPase (21). Numerous proteins have been reported to be 
transported by IPO8, including Smads (22), signal recognition particle protein 19 (21), and glucocorticoid receptor (23); however, the mechanisms leading to IPO 8 transcriptional activation remain elusive. To examine the mechanism underlying transcriptional regulation, the present study cloned and analyzed the 5 ' flanking segment of the IPO8 gene. Through a series of deletion studies, a crucial DNA fragment was identified at the 5' flanking promoter region (-732/-330 bp) responsible for maximum gene activation. Sequence analysis of the region between -732 and -330 bp identified a consensus sequence (TGTGGT) for RUNX2-binding sites $(16,17)$. Since RUNX2 is a bifunctional regulator that has the ability to activate or inhibit gene transcription based on the promoter context as well as cellular milieu, the RUNX2 binding site was mutated to investigate its effects on IPO 8 promoter activity. Mutation studies revealed that this RUNX2 binding site is required for maximum IPO 8 promoter activity. These data indicated that the RUNX2 binding site imparts a positive regulatory role in IPO 8 transcription. Confirmation of RUNX2 binding in vivo by ChIP indicated that RUNX2 binds to the critical promoter region of Saos- 2 cells, thus suggesting the physiological relevance of this DNA-protein interaction in IPO8 gene regulation.

The present study also investigated the effects of RUNX2 on IPO 8 expression. shRNA-mediated knockdown of RUNX2 induced a marked reduction in the mRNA expression of IPO 8 compared with that observed in the control cells, thus suggesting that the RUNX2 transcription factor has a positive role in regulating IPO 8 promoter activity.

Since RUNX2 acts as an upstream regulator for IPO8 transcription, it may be hypothesized that IPO 8 functions are associated with the function of RUNX2. The most significant function of RUNX2 is the control of osteoblast differentiation during the process of bone development. The present study demonstrated that IPO8 expression is synchronized with RUNX2 expression following osteoblast differentiation in Saos- 2 cells, which may be due to the fact that IPO 8 transcription is regulated by RUNX2. IPO 8 has long been considered a potential reference in gene expression investigations of human glioma, subcutaneous adipose tissues, and differentiated primary preadipocytes $(12,13)$. However, the results of the present study indicated that the degree of IPO8 expression may involve more variability that largely contributes to osteogenic differentiation following media stimulation. Until now, the association between IPO 8 and osteoblast differentiation has not yet been verified; however, some reports on IPOS function may provide indirect clues. As previously mentioned, translocation of Smad protein into the nucleus is mediated by IPO8. Smad proteins have previously been implicated as downstream effectors of transforming growth factor- $\beta$ /bone morphogenetic protein signaling $(24,25)$, which is the key pathway in the transcriptional control of bone formation. Furthermore, a previous study revealed that IPOS is required for AGO2 binding to a large series of target mRNAs and is necessary for miRNA-guided gene suppression (14). miRNAs are currently considered to be key regulators of a wide range of physiological and pathological processes, including cell proliferation, apoptosis, cancer, and osteogenic differentiation. It is therefore likely that the expression of IPO 8 has an unknown important role in cellular biology.
In conclusion, the findings of the present study provided novel insights into the control of IPOS transcription, and may enhance understanding regarding RUNX2 regulatory mechanisms in osteoblast differentiation, bone development, and degenerative bone disease. These results may assist in uncovering novel strategies of modulating IPO 8 gene expression, particularly during the design of treatment strategies for osteoporosis, bone repair, and other physiological processes.

\section{Acknowledgements}

The present study was supported by grants from the National Nature Science Foundation of China (grant no. 81260140 and 81360143) and the Key Projects Grant of Jiangxi Province Education Office (grant no. GJJ12683).

\section{References}

1. Bruder SP, Jaiswal N and Haynesworth SE: Growth kinetics, self-renewal, and the osteogenic potential of purified human mesenchymal stem cells during extensive subcultivation and following cryopreservation. J Cell Biochem 64: 278-294, 1997.

2. Engler AJ, Sen S, Sweeney HL and Discher DE: Matrix elasticity directs stem cell lineage specification. Cell 126: 677-689, 2006.

3. Geoffroy V, Kneissel M, Fournier B, Boyde A and Matthias P: High bone resorption in adult aging transgenic mice overexpressing cbfa1/runx 2 in cells of the osteoblastic lineage. Mol Cell Biol 22: 6222-6233, 2002.

4. Shui C, Spelsberg TC, Riggs BL and Khosla S: Changes in Runx2/Cbfa1 expression and activity during osteoblastic differentiation of human bone marrow stromal cells. J Bone Miner Res 18: 213-221, 2003.

5. Ducy P, Zhang R, Geoffroy V, Ridall AL and Karsenty G: Osf2/Cbfa1: A transcriptional activator of osteoblast differentiation. Cell 89: 747-754, 1997.

6. Teplyuk NM, Haupt LM, Ling L, Dombrowski C, Mun FK, Nathan SS, Lian JB, Stein JL, Stein GS, Cool SM and van Wijnen AJ: The osteogenic transcription factor Runx2 regulates components of the fibroblast growth factor/proteoglycan signaling axis in osteoblasts. J Cell Biochem 107: 144-154, 2009.

7. Stewart M: Molecular mechanism of the nuclear protein import cycle. Nat Rev Mol Cell Biol 8: 195-208, 2007.

8. Kutay U, Bischoff FR, Kostka S, Kraft R and Görlich D: Export of importin alpha from the nucleus is mediated by a specific nuclear transport factor. Cell 90: 1061-1071, 1997.

9. Cook A, Bono F, Jinek M and Conti E: Structural biology of nucleocytoplasmic transport. Annu Rev Biochem 76: 647-671, 2007.

10. Görlich D, Dabrowski M, Bischoff FR, Kutay U, Bork P Hartmann E, Prehn S and Izaurralde E: A novel class of RanGTP binding proteins. J Cell Biol 138: 65-80, 1997.

11. Nguewa PA, Agorreta J, Blanco D, Lozano MD, Gomez-Roman J, Sanchez BA, Valles I, Pajares MJ, Pio R, Rodriguez MJ, et al: Identification of importin 8 (IPO8) as the most accurate reference gene for the clinicopathological analysis of lung specimens. BMC Mol Biol 9: 103, 2008.

12. Kreth S, Heyn J, Grau S, Kretzschmar HA, Egensperger R and Kreth FW: Identification of valid endogenous control genes for determining gene expression in human glioma. Neuro Oncol 12: 570-579, 2010.

13. Hurtado del Pozo C, Calvo RM, Vesperinas-García G, Gómez-Ambrosi J, Frühbeck G, Corripio-Sánchez R, Rubio MA and Obregon MJ: IPO8 and FBXL10: New reference genes for gene expression studies in human adipose tissue. Obesity (Silver Spring) 18: 897-903, 2010.

14. Weinmann L, Höck J, Ivacevic T, Ohrt T, Mütze J, Schwille P, Kremmer E, Benes V, Urlaub $\mathrm{H}$ and Meister G: Importin 8 is a gene silencing factor that targets argonaute proteins to distinct mRNAs. Cell 136: 496-507, 2009.

15. Livak KJ and Schmittgen TD: Analysis of relative gene expression data using real-time quantitative PCR and the 2(-Delta Delta C(T)) Method. Methods 25: 402-408, 2001.

16. Komori T: Regulation of skeletal development by the Runx family of transcription factors. J Cell Biochem 95: 445-453, 2005. 
17. Little GH, Noushmehr H, Baniwal SK, Berman BP, Coetzee GA and Frenkel B: Genome-wide Runx2 occupancy in prostate cancer cells suggests a role in regulating secretion. Nucleic Acids Res 40: 3538-3547, 2012.

18. Komori T, Yagi H, Nomura S, Yamaguchi A, Sasaki K, Deguchi K Shimizu Y, Bronson RT, Gao YH, Inada M, et al: Targeted disruption of Cbfal results in a complete lack of bone formation owing to maturational arrest of osteoblasts. Cell 89: 755-764, 1997.

19. Nishio Y,Dong Y,Paris M, O'Keefe RJ, Schwarz EM and Drissi H: Runx2-mediated regulation of the zinc finger Osterix/Sp7 gene. Gene 372: 62-70, 2006.

20. van der Deen M, Akech J, Lapointe D, Gupta S, Young DW, Montecino MA, Galindo M, Lian JB, Stein JL, Stein GS and van Wijnen AJ: Genomic promoter occupancy of runt-related transcription factor RUNX2 in Osteosarcoma cells identifies genes involved in cell adhesion and motility. J Biol Chem 287: 4503-4517, 2012
21. Dean KA, von Ahsen O, Görlich D and Fried HM: Signal recognition particle protein 19 is imported into the nucleus by importin 8 (RanBP8) and transportin. J Cell Sci 114: 3479-3485, 2001.

22. Xu L, Yao X, Chen X, Lu P, Zhang B and Ip YT: Msk is required for nuclear import of TGF-\{beta\}/BMP-activated Smads. J Cell Biol 178: 981-994, 2007.

23. Freedman ND and Yamamoto KR: Importin 7 and importin alpha/importin beta are nuclear import receptors for the glucocorticoid receptor. Mol Biol Cell 15: 2276-2286, 2004.

24. Derynck R and Zhang YE: Smad-dependent and Smad-independent pathways in TGF-beta family signalling. Nature 425: 577-584, 2003

25. Retting KN, Song B, Yoon BS and Lyons KM: BMP canonical Smad signaling through Smad1 and Smad5 is required for endochondral bone formation. Development 136: 1093-1104, 2009. 